\title{
Juvenile idiopathic arthritis in relation to perinatal and maternal characteristics: a case control study
}

Samantha W. Bell ${ }^{1,45^{*}}$, Susan Shenoi ${ }^{2}$, J. Lee Nelson ${ }^{3}$, Parveen Bhatti ${ }^{1,3}$ and Beth A. Mueller ${ }^{1,3}$

\begin{abstract}
Background: Existing data on associations between maternal and early childhood exposures and juvenile idiopathic arthritis (JIA) risk is scant and inconsistent with previous studies showing potential role for prematurity, number of siblings and infections. We explored JIA and International League of Associations for Rheumatology (ILAR) JIA categories in relation to selected infant (birthweight, size-for-gestational-age, gestational age), and maternal (parity, delivery type, prior fetal loss) characteristics that may be markers for exposures related to two pathways (hygiene hypothesis, microchimerism) potentially associated with autoimmune disorder occurrence.

Methods: A case-control analysis with 1,234 JIA cases and 5,993 birth year-matched controls was conducted. Exposure information was obtained from WA state birth certificates. Multivariable logistic regression was used to estimate adjusted odds ratios (OR) and 95\% confidence intervals (Cl) for associations with maternal and early life exposures for JIA and JIA categories.

Results: Greater maternal parity was associated with a decreased OR for JIA (most marked for persistent oligoarticular $\mathrm{JIA}, \mathrm{OR} 0.32,95 \% \mathrm{Cl} 0.15 ; 0.71$, p for trend $=0.0001$ ). Prior fetal loss (except for oligoarticular JIA) was associated with an increased OR for JIA. Prematurity was associated with increased risk of enthesitis related arthritis (OR 1.9, 95\% Cl: 1.3-2.9) and rheumatoid factor positive polyarticular JIA (OR 2.2, 95\% Cl: 1.0-4.8).

Conclusions: We observed associations of selected maternal factors with JIA, some of which varied across JIA categories. The findings of decreased ORs for JIA in relation to greater maternal parity may be consistent with the hygiene and microchimerism hypotheses. Future studies with biomarkers relevant to these hypotheses will help elucidate any associations.
\end{abstract}

Keywords: Juvenile arthritis, Maternal reproductive history, Case-control study, Perinatal factors, Birth certificate

\section{Background}

Juvenile Idiopathic Arthritis (JIA) is a heterogeneous group of seven categories of chronic inflammatory arthritides in children with onset before 16 years of age [1]. JIA likely has a complex etiology, with multiple genetic and environmental factors contributing to its development [2]. Concordance rates of JIA among monozygotic twins are estimated at $25-40 \%$; suggesting that environmental factors may influence JIA risk [3].

\footnotetext{
* Correspondence: Samantha.Bell@ucsf.edu

'Department of Epidemiology, School of Public Health, University of Washington, Seattle, WA, USA

${ }^{4}$ School of Medicine, University of California, San Francisco, San Francisco, CA, USA

Full list of author information is available at the end of the article
}

Evidence suggests that adult rheumatoid arthritis (RA) [4], JIA [5, 6], and other autoimmune diseases such as multiple sclerosis $[7,8]$ may be associated with maternal or early childhood exposures such as maternal parity, birthweight, or gestational age.

Two hypotheses have been suggested relating perinatal and maternal reproductive characteristics to JIA. The "Hygiene hypothesis" suggests that early exposure to infection results in decreased risk of childhood autoimmune disease [9] due to modulation of the developing immune system. Proxy measures often used to assess this include maternal parity (a greater number of prior births being consistent with a greater number of older daycare- or school-aged siblings at home and greater 
possibility of early infection); infant birthweight, small size-for-gestational age, or gestational age (small, or preterm infants may be less resistant to infection), and mode of delivery (vaginal deliveries may more likely elicit a normal immune response due to early exposure to vaginal flora). The microchimerism hypothesis relates to maternal and/or sibling origin microchimerism (harboring of cells or DNA from another genetically distinct individual) from exchange of cells/DNA in utero from the mother or older siblings during gestation [10]. Microchimerism may potentially increase or decrease disease risk based on the specificity of the acquired genetic material from another individual [11], and some studies suggest that its presence may alter rheumatoid arthritis (RA) risk in women [12-17] although we could locate no studies of this in JIA. It has been suggested that the decreased RA risk in parous women may be due to microchimerism among women with prior births [12]. Proxy measures potentially associated with increased microchimerism may include greater maternal parity (greater likelihood that infant has acquired genetic material from older sibling(s)); plurality (twins and higher-order multiples may have acquired genetic material from their sibling in utero); and a maternal history of prior fetal loss (maternally-retained genetic material from a fetus may be passed to subsequent offspring).

Lacking direct indicators of early prior infection relevant to the hygiene hypothesis, or of the presence of microchimerism, we explored associations of JIA and its individual categories in relation to selected maternal reproductive and infant characteristics as proxies for these, as assessed by birth certificate data among children with, and without JIA. Elucidation of these relationships will enhance understanding of possible JIA risk factors.

\section{Methods \\ Study design}

This case-control study identified JIA cases at a regional children's hospital in the Seattle-Puget Sound region of Washington State using methods described previously [18]. Appropriate Institutional Review Board approvals were obtained. Potential JIA cases were identified though International Classification of Diseases (ICD-9), Ninth Revision diagnosis codes (720.0, 696.0, 714.32, 714.31, 714.30, 720.89, 714.33, 714.3) from $1997-2010(N=2,274)$ and linked to WA State birth records to identify children born in-state $(N=1,518)$. Controls were randomly selected in a $4: 1$ control: case ratio from remaining birth records, matched on year of birth $(N=6,072)$.

Chart review further refined JIA identification and categorized cases into ILAR categories including: polyarticular rheumatoid factor (RF) positive, polyarticular RF negative, oligoarticular arthritis (persistent and extended), psoriatic JIA, enthesitis related arthritis, systemic JIA, and undifferentiated JIA. We excluded 228 cases who did not meet ILAR criteria [1], and 38 cases with disease onset at $>16$ years, resulting in 1,252 cases for analyses. After examining the OR for JIA in relation to plurality status, all remaining analyses were restricted to singleton infants only (1,234 cases; 5,993 controls) because characteristics (for example, birthweight and gestational age) of infants and pregnancies differ for singleton and multiple gestation pregnancies.

Exposure information from birth records included: birthweight $(<2500,2500-<4000,4000+$ grams $)$; sizefor-gestational-age (SGA; small, AGA; appropriate, or LGA; large, based on above/below the 10th percentile for size/gestational age in Washington birth record standards); and gestational age $(<37,37-<42,42+$ weeks). Maternal and infant factors relevant to the hygiene hypothesis included: number of prior births; delivery method (vaginal, cesarean (C) section), infant birthweight, gestational age and size for gestational age. To examine the microchimerism hypothesis, we examined birth order (as measured by maternal parity), plurality (singleton, twin/multiple gestation), and number of prior fetal losses (any; and by <20, 20+ weeks gestation).

\section{Statistical analysis}

Multivariable logistic regression was used to estimate odds ratios (OR) and 95\% confidence intervals (CIs) for associations, adjusted for maternal age, and the matching variable birth year. Other factors evaluated for possible effects on the relationships of interest included paternal age; maternal race/ethnicity, educational level, prenatal smoking, and marital status; medical insurance at birth (Medicaid vs. other); sex; and trimester prenatal care began. We adjusted for factors that altered the OR by greater than $10 \%$ in univariate analyses. Potential effect modifiers were evaluated by inspection of stratum-specific ORs and the Breslow-Day test for homogeneity. Trends were evaluated using Wilcoxon rank sum test. Analyses were initially conducted to examine JIA overall, and repeated for JIA categories (restricted to factors with at least 5 subjects in a cell). Analyses were conducted using Stata (version 11; StataCorp, College Station, Texas).

\section{Results}

The majority of cases $(67 \%)$ were female. Mothers of cases' were more likely to be white $(81 \%)$, married $(80 \% \mathrm{v})$, college graduates $(34 \%)$, have first trimester prenatal care $(86 \%)$ and less likely to have Medicaid insurance (26\%) (Table 1). Maternal gravidity was similar among cases and controls, with about $30 \%$ of mothers being nulliparous. Distribution of JIA categories included: 
Table 1 Infant and Maternal Characteristics of JIA Cases and non-JIA Controls born in Washington State 1997-2010

\begin{tabular}{|c|c|c|c|c|}
\hline \multirow[t]{2}{*}{ Characteristics } & \multicolumn{2}{|c|}{$\begin{array}{l}\text { Cases } \\
(N=1,252)^{a}\end{array}$} & \multicolumn{2}{|c|}{$\begin{array}{l}\text { Controls } \\
(N=6,072)^{a}\end{array}$} \\
\hline & $\mathrm{n}$ & $\%$ & $\mathrm{n}$ & $\%$ \\
\hline \multicolumn{5}{|l|}{ Infant Factors } \\
\hline Female & 843 & 67.3 & 2,903 & 47.8 \\
\hline Twin/multiple gestation $^{\mathrm{b}}$ & 18 & 1.4 & 79 & 1.3 \\
\hline \multicolumn{5}{|l|}{ Race/Ethnicity } \\
\hline White & 984 & 81.3 & 4,425 & 74.8 \\
\hline Black & 37 & 3.1 & 316 & 5.3 \\
\hline Asian/Pacific Islander & 60 & 4.9 & 426 & 7.2 \\
\hline Native American & 38 & 3.1 & 150 & 2.5 \\
\hline Hispanic & 91 & 7.5 & 598 & 10.1 \\
\hline Other & 1 & 0.1 & 3 & 0.1 \\
\hline \multicolumn{5}{|l|}{ Maternal Factors } \\
\hline \multicolumn{5}{|l|}{ Age (years) } \\
\hline$<20$ & 106 & 8.5 & 694 & 11.4 \\
\hline $20-24$ & 233 & 18.6 & 1,536 & 25.3 \\
\hline $25-29$ & 385 & 30.8 & 1,774 & 29.2 \\
\hline $30-34$ & 341 & 27.2 & 1,326 & 21.9 \\
\hline $35+$ & 187 & 14.9 & 739 & 12.2 \\
\hline \multicolumn{5}{|l|}{ Education (years) } \\
\hline$<12$ & 100 & 12.9 & 639 & 18.0 \\
\hline 12 & 227 & 29.3 & 1,092 & 30.8 \\
\hline $12-16$ & 188 & 24.2 & 927 & 26.1 \\
\hline $16+$ & 261 & 33.6 & 888 & 25.0 \\
\hline Unmarried & 250 & 20.0 & 1,577 & 26.1 \\
\hline \multicolumn{5}{|c|}{ Trimester of inception of prenatal care } \\
\hline First & 987 & 85.9 & 4,591 & 81.1 \\
\hline Second & 133 & 11.6 & 897 & 15.8 \\
\hline Third & 29 & 2.5 & 175 & 3.1 \\
\hline Medicaid insurance & 282 & 26.3 & 1,789 & 37.0 \\
\hline
\end{tabular}

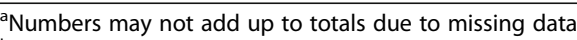

${ }^{\mathrm{b}} \mathrm{OR}$ for association with $\mathrm{JIA}=1.04,95 \% \mathrm{Cl}$ : 0.62-1.74

oligoarticular; persistent (31.5\%) and extended (5.9\%), enthesitis related arthritis (21.2\%), RF negative polyarticular (20.7\%), systemic arthritis (5.8\%), psoriatic arthritis (5.0\%), RF positive polyarticular arthritis (4.9\%), and undifferentiated arthritis (3.2\%). The remaining $1.8 \%$ was polyarticular arthritis with unknown RF status.

\section{Infant and maternal characteristics in relation to JIA}

Multiple gestation pregnancy was not associated with JIA (Table 1); OR 1.04, 95\% CI: 0.62-1.74). Among singletons, birthweight $>4000 \mathrm{~g}$ was associated with a decreased OR for JIA (OR 0.81, 95\% CI: 0.67-0.98) (Table 2). The OR for JIA decreased with increasing number of prior maternal deliveries, with the lowest OR observed for children of women with two prior deliveries (0.68, 95\% CI: $0.56-0.83$ for 2 deliveries, $p$ for trend $=0.0007)$. No associations of JIA were observed with gestational age, size-for-gestational age, $\mathrm{C}$-section delivery or maternal history of prior fetal losses.

\section{Infant and maternal characteristics in relation to individual JIA categories}

We observed an increased ORs for prematurity and RF+ polyarthritis (OR 2.23, 95\% CI: 1.04-4.80) and enthesitisrelated arthritis (OR 1.90, 95\% CI: 1.27-2.86) (Table 3). SGA was associated with increased risk of undifferentiated JIA (OR 2.64, 95\% CI: 1.18-5.94). Birthweight <2500 grams was associated with RF+ polyarthritis (OR 2.29, 95\% CI: 1.03-5.13).

The pattern we observed for overall JIA of decreasing ORs in relation to increasing number of maternal prior deliveries was marked for persistent oligoarthritis, with a monotonically decreasing trend ranging from 0.88 (95\% CI: $0.69-1.12)$ for one prior birth, to 0.32 (95\% CI: $0.15-0.71$ ) for $4+$ prior deliveries ( $\mathrm{p}$ for trend $=0.0001$ ), and a suggestion of trend for extended oligoarthritis. Non-statistically significant decreased ORs were observed for greater numbers of prior deliveries for most other JIA categories, except for RF- (OR 1.24, 95\% CI: $0.70-2.18$ for $4+$ prior deliveries) and RF+ (OR 1.77, 95\% CI: $0.75-4.14$ for $3+$ prior deliveries) polyarthritis.

ORs were increased in relation to maternal fetal loss for psoriatic JIA (OR 2.85, 95\% CI: 1.57-5.17); RF- polyarthritis (OR 2.20, 95\% CI: 1.55-3.12); RF+ polyarthritis (OR 2.00, 95\% CI: 1.00-4.00); systemic JIA (OR 1.90, 95\% CI: 1.00-3.64) and enthesitisrelated arthritis (OR 1.59, 95\% CI: 1.16-2.17). Increased ORs were noted with maternal history of early fetal loss $(<20$ weeks) for enthesitis-related arthritis (OR 1.56, 95\% CI: 1.13-2.14), RF- polyarthritis (OR 2.08, 95\% CI: 1.46-2.96), and psoriatic (OR 2.62, 95\% CI: 1.44-4.79).

\section{Discussion}

We observed a decreased risk of JIA overall in relation to greater maternal parity, with a significant monotonic trend for persistent oligoarthritis, and suggestion of decreased ORs in relation to increasing maternal parity for most other JIA categories. This finding may be consistent with the hygiene hypotheses as children with older siblings likely have greater opportunity for infection exposure, both in utero and during post-natal life. Additionally a modest, non-statistically significant increased risk of JIA with Cesarean delivery similar to a Swedish registry report [5] provides weak support for the hygiene hypothesis, although this has not been supported in all studies [4]. 
Table 2 Infant and maternal characteristics among singleton Juvenile Idiopathic Arthritis (JIA) cases and controls born in WA State, 1997-2010

\begin{tabular}{|c|c|c|c|c|c|c|}
\hline \multirow[t]{2}{*}{ Characteristics } & \multicolumn{2}{|c|}{ Cases $(N=1,234)$} & \multicolumn{2}{|c|}{ Controls $(N=5,993)$} & \multirow[b]{2}{*}{$\mathrm{OR}^{\mathrm{a}}$} & \multirow[b]{2}{*}{$95 \% \mathrm{Cl}$} \\
\hline & $\mathrm{n}$ & $\%$ & $\mathrm{~N}$ & $\%$ & & \\
\hline \multicolumn{7}{|l|}{ Infant factors } \\
\hline \multicolumn{7}{|c|}{ Gestational age (weeks) } \\
\hline$<37$ & 72 & 6.0 & 352 & 6.0 & 0.99 & $0.76,1.29$ \\
\hline $37-42$ & 1,030 & 85.5 & 4,903 & 83.9 & 1.00 & reference \\
\hline $42+$ & 102 & 8.5 & 587 & 10.1 & 0.88 & $0.70,1.10$ \\
\hline \multicolumn{7}{|c|}{ Size for gestational age } \\
\hline SGA & 102 & 9.4 & 496 & 9.6 & 1.01 & $0.81,1.27$ \\
\hline AGA & 878 & 80.5 & 4,181 & 80.8 & 1.00 & reference \\
\hline LGA & 110 & 10.1 & 500 & 9.6 & 1.01 & $0.81,1.26$ \\
\hline \multicolumn{7}{|c|}{ Birth weight (grams) } \\
\hline$<2500$ & 64 & 5.2 & 292 & 4.9 & 1.05 & $0.79,1.39$ \\
\hline 2500-3999 & 1,026 & 83.3 & 4,880 & 81.7 & 1.00 & reference \\
\hline$\geq 4000$ & 142 & 11.5 & 803 & 13.4 & 0.81 & $0.67,0.98$ \\
\hline \multicolumn{7}{|l|}{ Maternal factors } \\
\hline \multicolumn{7}{|c|}{ Cesarean Section } \\
\hline Yes & 256 & 20.7 & 1,110 & 18.5 & 1.08 & $0.93,1.26$ \\
\hline No & 978 & 79.3 & 4,878 & 81.5 & 1.00 & reference \\
\hline \multicolumn{7}{|c|}{ Number of prior births ${ }^{b}$} \\
\hline 0 & 525 & 43.6 & 2,488 & 42.5 & 1.00 & reference \\
\hline 1 & 418 & 34.7 & 1,893 & 32.3 & 0.95 & $0.82,1.10$ \\
\hline 2 & 157 & 13.0 & 931 & 15.9 & 0.68 & $0.56,0.83$ \\
\hline 3 & 60 & 5.0 & 325 & 5.6 & 0.71 & $0.53,0.96$ \\
\hline $4+$ & 44 & 3.7 & 216 & 3.7 & 0.72 & $0.51,1.02$ \\
\hline \multicolumn{7}{|c|}{ Any fetal loss (early or late) } \\
\hline None & 482 & 67.0 & 2,388 & 67.4 & 1.00 & reference \\
\hline $1+$ & 237 & 33.0 & 1,154 & 32.6 & 0.99 & $0.84,1.18$ \\
\hline \multicolumn{7}{|c|}{ Number of prior fetal losses $(<20 \text { weeks })^{c}$} \\
\hline None & 500 & 69.5 & 2,472 & 69.8 & 1.00 & reference \\
\hline $1+$ & 219 & 30.5 & 1,069 & 30.2 & 0.99 & $0.83,1.18$ \\
\hline \multicolumn{7}{|c|}{ Number of prior fetal losses ( $\geq 20$ weeks) ${ }^{c, d}$} \\
\hline None & 698 & 97.1 & 3,429 & 97.1 & 1.00 & reference \\
\hline $1+$ & 21 & 2.9 & 104 & 2.9 & 1.09 & $0.67,1.77$ \\
\hline
\end{tabular}

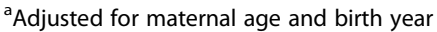

${ }^{\mathrm{b}} \mathrm{p}$ for trend ${ }^{* * * * P} 0.0007$

'Restricted to 839 cases and 4,015 control whose mothers had a prior pregnancy

${ }^{\mathrm{d}}$ Adjusted for maternal age, birth year, and paternal age

Consistent with our data, a previous Australian clinicbased study showed that having any siblings (vs. none) was protective for JIA in (OR 0.46, 95\% CI 0.28; 0.74, using clinic-based controls); a result slightly attenuated (OR 0.50, 95\% CI 0.24; 1.02) when community controls were used [10]. An OR of 1.6 for JIA was observed in relation to being an only child in a Danish registry-based study [19]. In contrast, no risk reduction was observed in relation to having increased numbers of older siblings in a Swedish registry-based study [5]. Birth order was not found to be associated with JIA in two previous studies $[6,20]$.

The presence of microchimerism might increase or decrease JIA risk based on the nature of the acquired genetic material. An inverse association of JIA with greater maternal parity may be related to the presence of 


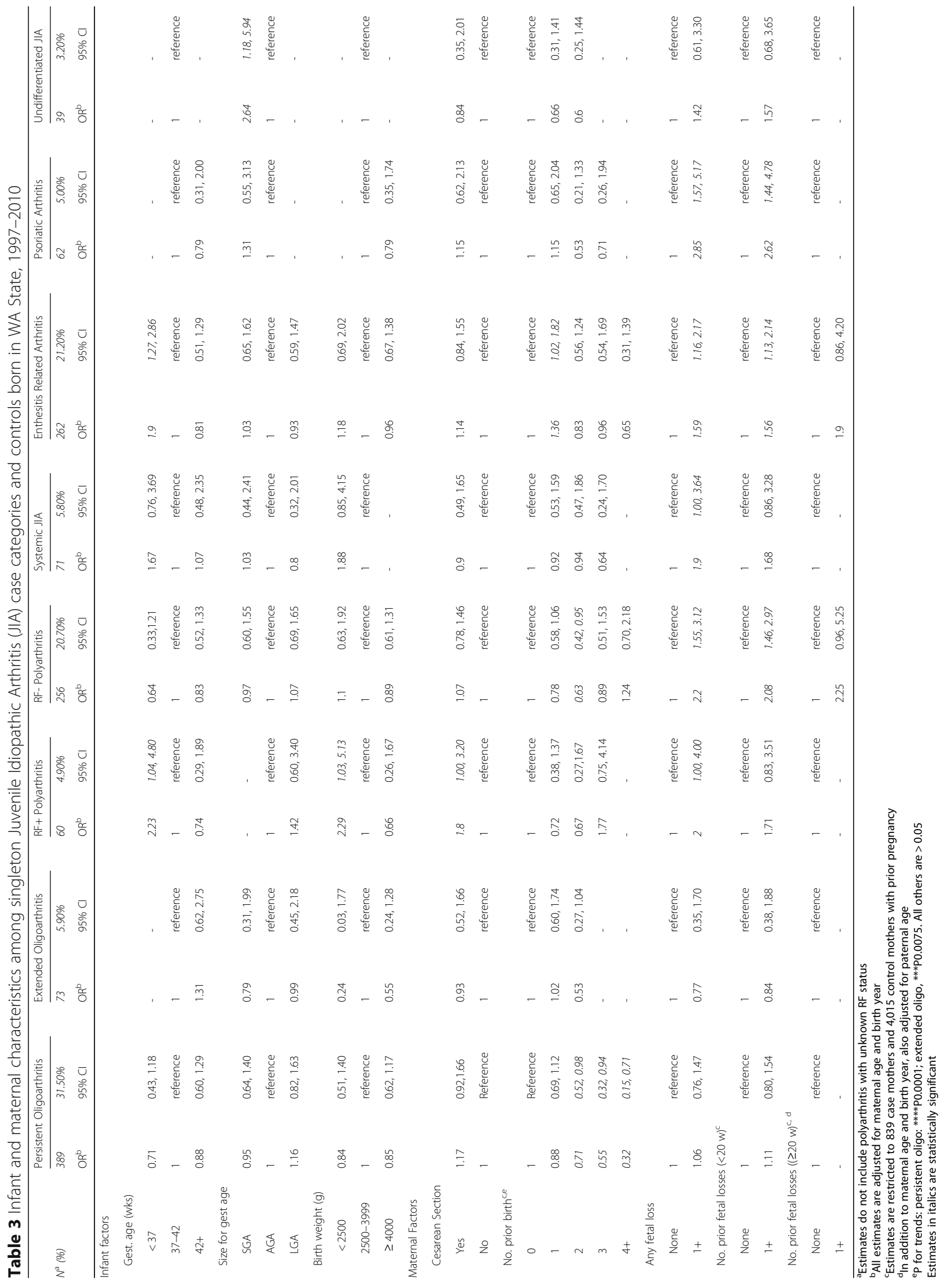


acquired genetic material carrying JIA-protective Human Leukocyte Antigen (HLA) alleles from an older sibling. In our study, most JIA category-specific risk estimates for maternal fetal loss were increased; a finding that could be consistent with the microchimerism hypothesis only if presence of increased levels of acquired microchimerism from a mother's prior pregnancy carried JIA-associated risk HLA alleles [11]. However, it has been demonstrated that mothers of children with JIA have impaired reproductive capacity [21], so history of maternal fetal loss may also simply be an indicator of this observed association. Ultimately, we had no information about specific gene traits of children or their families, and this is speculation.

In contrast with previous studies from Sweden and United States [5, 6], high birthweight in our study was associated with a significantly decreased risk of JIA overall. Reasons for this difference are not clear.

Previous studies related to a gestational age effect vary. Although Swedish registry data suggest a borderline decreased OR in relation to preterm delivery [5], Shenoi et al [6] noted a significantly increased risk of JIA in relation to prematurity. While our results suggest no association with gestational age overall, enthesitis-related arthritis and RF+ polyarticular JIA had significantly increased ORs in relation to preterm gestation. Mothers of children with JIA have higher rates of preterm delivery [21] and the association of prematurity with JIA needs to be further explored in future studies.

The use of outpatient and inpatient medical records maintained by board-certified pediatric rheumatologists at a large regional children's medical center, with data linkage to state birth records, are major strengths of this study enabling identification of a relatively large number of cases. Exposure information was assessed from birth records prior to onset and diagnosis of JIA, and was not subject to recall bias. Because of the known polygenic genetic predispositions and heterogeneity of the pathogenesis of differing JIA categories, it is important to examine these relationships separately for each category; to our knowledge, this is the first study to do so. Medical chart review allowed us to confirm and ascertain JIA categories, thereby reducing misclassification.

Some limitations of our study include possible inaccuracies in reporting of mother's reproductive history; in particular, inaccurate reporting of fetal loss on the birth certificates may have lead to nondifferential misclassification, biasing the OR estimates towards the null. Birthweight and number of prior live births, however, have been assessed as reliable in comparison to medical records [22]. There is potential for unmeasured confounding due to unrecognized pregnancy loss, psychosocial support factors, and lack of information on fertility, or residual confounding due to the necessity of reliance on birth records for exposure information. The inability to measure these could lead to residual confounding in our risk estimates and could bias the estimates either way. Although we attempted to identify all children with JIA at the sole facility with boardcertified pediatric rheumatologists in Washington State during the study period, and free care is available for families without resources, it is possible that our JIA cases may not be representative of all children with JIA in Washington State. Some cases may have been treated by adult rheumatologists or other practitioners outside this facility, or they may differ from the underlying population in ways that we could not measure. We attempted to evaluate any related bias due to this, although adjustment for socio-economic and other factors potentially related to care access had no effect on the results. Our inability to examine the presence of younger siblings is also a limitation.

\section{Conclusions}

We observed decreased JIA risk with increasing parity, which potentially supports the hygiene or microchimerism hypotheses, with consistent results demonstrated in many JIA categories. Of note, our results indicate the need to consider JIA categories as separate entities; indeed our results were often inconsistent across categories, suggesting potentially different etiologies. Given the current lack of preventative or curative treatments for JIA, further studies, particularly those capable of examining category-specific JIA associations, are warranted. A better understanding of these associations will help inform prevention programs and hopefully reduce the burden of this debilitating disease.

\section{Abbreviations \\ Cl: Confidence interval; ICD-9: International classification of diseases;} JIA: Juvenile idiopathic arthritis; OR: odds ratio; RF: Rheumatoid factor

\section{Acknowledgements}

We would like to thank the Washington State Department of Health for data access, and Mr. Bill O’Brien for programming assistance.

\section{Funding}

This project was supported in part by the Health Resources and Services Administration's Maternal and Child Health Bureau (Title V, Social Security Act), grant \# T76MC00011-21-00. Dr Shenoi's work was supported by an NIH T32AR007108 grant.

\section{Availability of data and material}

The data that support the findings of this study are available from the Washington State IRB but restrictions apply to the availability of these data, which were used under license for the current study, and so are not publicly available. Data are however available from the authors upon reasonable request and with permission of the Washington State IRB.

\section{Authors' contributions}

All authors were involved in drafting the article or revising it critically for important intellectual content, and all authors approved the final version to be submitted for publication. Ms. SWB had full access to all 
of the data in the study and takes responsibility for the integrity of the data and the accuracy of the data analysis. Study conception and design. SWB, BAM, SS, PB. Acquisition of data. SS, SWB, BAM. Analysis and interpretation of data. SS, SWB, BAM, JLN.

\section{Competing interests}

The authors declare that they have no competing interests.

\section{Consent for publication}

Not applicable.

\section{Ethics approval and consent to participate}

This study was approved by Institutional Review Boards for Protection of Human Subjects of Washington State and Seattle Children's Hospital (SCH).

\section{Publisher's Note}

Springer Nature remains neutral with regard to jurisdictional claims in published maps and institutional affiliations.

\section{Author details}

'Department of Epidemiology, School of Public Health, University of Washington, Seattle, WA, USA. ${ }^{2}$ Department of Pediatrics, Division of Rheumatology, Seattle Children's Hospital, University of Washington School of Medicine, Seattle, WA, USA. ${ }^{3}$ Public Health Sciences Division, Fred Hutchison Cancer Research Center, Seattle, WA, USA. ${ }^{4}$ School of Medicine, University of California, San Francisco, San Francisco, CA, USA. ${ }^{5}$ University of California, San Francisco, 513 Parnassus Ave., Room S245, San Francisco, CA 94143, USA.

\section{Received: 17 February 2017 Accepted: 1 May 2017}

\section{Published online: 11 May 2017}

\section{References}

1. Petty RE, Southwood TR, Manners P, et al. International league of associations for rheumatology classification of juvenile idiopathic arthritis: Second revision, edmonton, 2001. J Rheumatol. 2004;31(2):390-2.

2. Cassidy J, Petty R, Laxer R, Lindsley C. Textbook of pediatric rheumatology: Expert consult. Vol 6th ed. 2011.

3. Ellis JA, Munro JE, Ponsonby AL. Possible environmental determinants of juvenile idiopathic arthritis. Rheumatology (Oxford). 2010;49(3):411-25.

4. Colebatch AN, Edwards CJ. The influence of early life factors on the risk of developing rheumatoid arthritis. Clin Exp Immunol. 2011;163(1):11-6.

5. Carlens C, Jacobsson L, Brandt L, Cnattingius S, Stephansson O, Askling J. Perinatal characteristics, early life infections and later risk of rheumatoid arthritis and juvenile idiopathic arthritis. Ann Rheum Dis. 2009;68(7):1159-64.

6. Shenoi S, Shaffer ML, Wallace CA. Environmental risk factors and early-life exposures in juvenile idiopathic arthritis: A case-control study. Arthritis Care Res (Hoboken). 2016:68(8):1186-94.

7. Mueller BA, Nelson JL, Newcomb PA. Intrauterine environment and multiple sclerosis: A population- based case-control study. Mult Scler. 2013;19(1):106-11.

8. Nijland MJ, Ford SP, Nathanielsz PW. Prenatal origins of adult disease. Curr Opin Obstet Gynecol. 2008;20(2):132-8.

9. Bach JF. The effect of infections on susceptibility to autoimmune and allergic diseases. N Engl J Med. 2002;347(12):911-20.

10. Miller J, Ponsonby AL, Pezic A, et al. Sibling exposure and risk of juvenile idiopathic arthritis. Arthritis Rheumatol. 2015;67(7):1951-8.

11. Nelson JL. The otherness of self: Microchimerism in health and disease. Trends Immunol. 2012;33(8):421-7.

12. Guthrie KA, Gammill HS, Madeleine MM, Dugowson CE, Nelson JL. Parity and HLA alleles in risk of rheumatoid arthritis. Chimerism. 2011;2(1):11-5.

13. Kekow M, Barleben M, Drynda S, Jakubiczka S, Kekow J, Brune T. Long-term persistence and effects of fetal microchimerisms on disease onset and status in a cohort of women with rheumatoid arthritis and systemic lupus erythematosus. BMC Musculoskelet Disord. 2013;14:325.

14. Rak JM, Maestroni L, Balandraud N, Guis S, Boudinet H, Guzian MC, Yan Z, Azzouz D, Auger I, Roudier C, Martin M, Didelot R, Roudier J, Lambert NC. Transfer of the shared epitope through microchimerism in women with rheumatoid arthritis. Arthritis Rheum. 2009;60(1):73-80.

15. Yan Z, Aydelotte T, Gadi VK, Guthrie KA, Nelson JL. Acquisition of the rheumatoid arthritis HLA shared epitope through microchimerism. Arthritis Rheum. 2011;63(3):640-4
16. Feitsma AL, Worthington J, van der Helm-van Mil A, Plant D, Thomson W, Ursum J, van Schaardenburg D, van der Horst-Bruinsma I, van Rood JJ, Huizinga T, Toes R, de Vries RRP. Protective effect of noninherited maternal HLA-DR antigens on rheumatoid arthritis development. Proc Natl Acad Sci. 2007;104:19966-70. USA.

17. Stevens AM. Maternal microchimerism in health and disease. Best Pract Res Clin Obstet Gynaecol. 2016;31:121-30 (review).

18. Shenoi S, Bell S, Wallace CA, Mueller BA. Juvenile idiopathic arthritis in relation to maternal prenatal smoking. Arthritis Care Res (Hoboken). 2015; 67(5):725-30.

19. Nielsen HE, Dorup J, Herlin T, Larsen K, Nielsen S, Pedersen FK. Epidemiology of juvenile chronic arthritis: Risk dependent on sibship, parental income, and housing. J Rheumatol. 1999;26(7):1600-5.

20. Prahalad S, Fraser AM, O'Brien E, Kerber RA, Mineau GP, Bohnsack JF. Lack of association between birth order and juvenile idiopathic arthritis. Arthritis Rheum. 2003;48(10):2989-90.

21. Chaudhari M, Moroldo MB, Shear E, et al. Impaired reproductive fitness in mothers of children with juvenile autoimmune arthropathies. Rheumatology (Oxford). 2006;45(10):1282-7.

22. Roohan PJ, Josberger RE, Acar J, Dabir P, Feder HM, Gagliano PJ. Validation of birth certificate data in new york state. J Community Health. 2003;28(5):335-46.

\section{Submit your next manuscript to BioMed Central and we will help you at every step:}

- We accept pre-submission inquiries

- Our selector tool helps you to find the most relevant journal

- We provide round the clock customer support

- Convenient online submission

- Thorough peer review

- Inclusion in PubMed and all major indexing services

- Maximum visibility for your research

Submit your manuscript at www.biomedcentral.com/submit 